\title{
THE TURNPIKE OF DYNAMIC GENERAL EQUILIBRIUM PATHS AND ITS INSENSITIVITY TO INITIAL CONDITIONS*
}

\author{
Makoto YANO \\ Cornell University, Ithaca, NY 14853, USA
}

Received November 1983, accepted July 1984

\begin{abstract}
Bewley considers a dynamic general equilibrium model with heterogeneous consumers. Assuming that the future is sufficiently important, he shows that an equilibrium path converges to a limit which depends upon initial conditions. This study shows that the limit lies near the stationary state independent of initial conditions. This state is the economy's so-called turnpike - a turnpike is usually considered to be independent of initial conditions. An equilibrium path exists only if consumers discount future utilities. Despite this myopia of consumers, dynamic equilibria turn out to be almost Pareto efficient with respect to the non-myopic preferences defined by consumers' periodwise utility functions.
\end{abstract}

\section{Introduction}

In his seminal paper, Bewley (1982) applies capital theory to general equilibrium theory. He considers a model with infinitely many periods and perfect future markets where transactions are taking place among infinitely lived heterogeneous agents who discount the future. He shows that a dynamic general equilibrium (GE) path in this decentralized market model converges as time passes, if the future is sufficiently important. As he points out in the introduction of his paper, the limit of an equilibrium path depends upon initial conditions. In contrast, in the traditional turnpike theorems it is shown that there is a path (turnpike) with the following two characteristics [McKenzie (1976)]:

(1) any optimal paths stay within a small neighborhood of the turnpike almost all the time,

(2) the turnpike is independent of initial conditions (and terminal conditions if a finite time horizon model is considered).

Since the result of Bewley lacks the second characteristic above, it is weaker than the traditional theorems.

\footnotetext{
*This is based upon part of Chapter 3 of my dissertation submitted to the University of Rochester. I would like to thank my advisers, Professors R. Jones and L. McKenzie for their encouragement. When writing the chapter, I was especially indebted to Professor McKenzie for his guidance. Also I would like to thank C. Bates, W. Brock, J. Friedman, and P. Weller for comments.
} 
The first result of this study is that the dynamic GE model also has a turnpike with both characteristics (1) and (2). We will show that for any initial condition and any positive $\varepsilon$, there is a period such that an equilibrium path satisfying the initial condition stays within the $\varepsilon$ neighborhood of the turnpike after the period, as long as the future is sufficiently important. The allocation which is repeated every period on the turnpike is characterized as the equilibrium of an ordinary static general equilibrium model. This model is independent of initial conditions and has the single period technology and utility functions upon which the dynamic GE model is based [see Bewley (1982, eq. (1.18))]. The intuitive reason for our turnpike theorem is the following: If the future becomes more and more important, the temporary component of each consumer's wealth, or the value of his initial capital, becomes negligible since the permanent component of his wealth, or the present value of the stream of his primary good endowments over the future, becomes closer and closer to infinity. Thus, the role of an initial allocation of capital in determining an equilibrium also becomes negligible.

The second result of this study is related to efficiency. In order to define an equilibrium path, we assume that a consumer, say, consumer $i$ has the preference relation which is represented by the discounted sum of his utility functions, $\sum_{t=1}^{\infty} \rho^{t} u^{i}\left(X_{t}\right)$, where $0<\rho<1$. An equilibrium path is, therefore, Pareto efficient with respect to their preference relations which reflect the future discounting. It is often considered, however, that discounting the future should be attributed to the myopia of consumers, which is undesirable. Thus, it is worthwhile evaluating an equilibrium path with respect to preference relations which do not reflect their myopia but are based upon their tastes. For this purpose, we consider the following preference relation. That is, a consumption path $\left\{x_{t}^{\prime}\right\}$ is preferred to another path $\left\{x_{t}\right\}$ if $\limsup \sup _{T \rightarrow \infty} \sum_{t=1}^{T}\left(u^{i}\left(x_{t}\right)-u^{i}\left(x_{t}^{\prime}\right)\right)<0$. We call this preference relation a nonmyopic preference. It is shown that an equilibrium path is almost Pareto efficient with respect to non-myopic preferences in spite of the myopia of consumers. As a direct corollary of this result, it is shown that the turnpike is Pareto efficient with respect to non-myopic preferences.

Our turnpike theorem has two important implications which Bcwlcy's theorem does not have. First, it provides a simple test of the market imperfection of an economy which does not have perfect future markets. Second, the turnpike theorem provides a dynamic foundation for static GE models.

We do not claim that our dynamic GE model is realistic. The model is useful, however, as a standard with which more realistic models of intertemporal allocation can be compared. Since our markets are purely perfect, the efficiency, which we consider for a dynamic GE path, is that with respect to one of the strictest standards. If one has to deal with an economy with 
imperfect future markets in reality, he may test the degree of market imperfection by comparing an intertemporal allocation in such a model with a dynamic GE path. An explanation along a similar line is often accepted as a reason why we study sophisticated GE models. If one, however, intends to use our dynamic GE model to test market imperfection, one has to face an extremely difficult computational problem specific to our model: first, solving an infinite dimensional optimization problem for each agent and for each sequence of prices from the present to the future and, second, finding a solution to the resulting infinite dimensional simultaneous equation system of excess demand functions. This computational procedure suggests that even if solutions are obtained, they are at best approximations. Our turnpike theorem provides us with a test of the degree of market imperfection where we may avoid this difficult computational problem. That is, we may consider that an intertemporal allocation in an economy with imperfect future markets is almost efficient (is not efficient) if it is close to (is far away from) the turnpike. Since the turnpike approximates GE paths, this test provides an approximated result.

Static GE models are used in many fields of applied economic theory. An equilibrium in such a model is considered to abstract a state after every possible adjustment is completed. This intuitive explanation is given frequently in basic textbooks in answering the question of why we study static GE models. Moreover, in order to explain this intuition rigorously, many adjustment processes, including the tatonnement, have been considered. However, such a process is based upon a descriptive adjustment mechanism (such as that of the tatonnement) and almost always, if not always, completely ignores the intertemporal decision making of economic agents. In contrast, we consider a model where intertemporal optimization behaviors play a central role. Our result shows that the long-run state of our dynamic GE model is approximately characterized by an equilibrium in an ordinary static GE model which is independent of initial conditions. Analysing a static GE model may therefore be considered to be studying the long-run state of a dynamic economy where each agent optimizes intertemporally with perfect foresight. (Of course, this is not true in a strict sense unless assumptions such as ours are satisfied.) This shows that the optimization behaviors of agents alone can give a rationale to static GE models where heterogenous consumers are considered.

This study is based upon the dynamic GE model of Yano (1984), where results similar to the Bewley's are shown under a set of weaker assumptions. The main results of this study, however, also hold in Bewley's framework.

Mathematical Notation. Before we start describing our model, some mathematical notation should be clarified. $R^{J}$ is the Cartesian product of $J$ real lines; $R_{+}^{J}$ is its non-negative orthant; $|\cdot|$ is the Euclidian norm on $R^{J}$. A 
sequence in $R^{J}$ is denoted as $\underset{\sim}{x}$. For a vector $x, x \geqq 0$ means that $x_{j} \geqq 0$ for all $j ; x \gg 0$ that $x_{j}>0$ for all $j ; x>0$ that $x \geqq 0$ and $x \neq 0 . \chi^{\bar{A}}$ is the characteristic function from the set of all subsets of $\bar{J}$ to $R^{J}$; i.e., for $\bar{A} \subset \bar{J}, \chi_{j}^{\bar{A}}=1$ if $j \in \bar{A}$ and $=0$ if $j \notin \bar{A}$. For $a \in R^{J}$ and $X \subset R^{J}, a+X=\{a+x: x \in X\}$.

\section{Model}

(1). The time horizon of the economy is infinite.

(2) Commodities. At each period $t=1,2, \ldots$, there are $J$ commodities. $\bar{J}=$ $\{1,2, \ldots, J\}$. The commodity space is $R^{J} . \bar{P}=\{1, \ldots, P\}$ is the set of producible goods and $\overline{0}=\{j \in \bar{J}: j \notin \bar{P}\}$ is the set of primary goods. $\bar{C}^{i}$ is the set of consumption goods of consumer $i$.

(3) Commodity space. For $x \in R^{J}$, we define $x^{P} \in R^{J}$ as follows. $x_{j}^{P}=x_{j}$ if $j \in \bar{P}$ and $x_{j}^{P}=0$, otherwise. Let $R^{P}=\left\{x^{P} \in R^{J}: x \in R^{J}\right\}$ and $R_{+}^{P}=\left\{x^{P} \in R^{P}: x \in R_{+}^{J}\right\}$. In the same way, we define $x^{0}, R^{0}, x^{C^{i}}$ and so on.

(4) Technology. A social production set, $Y$, relates capital input of the previous period to a production activity of the present period. $Y$ is a closed convex cone in $R^{P} \times R^{J}$. $(-k, y) \in Y$ means that if $k \geqq 0$ was kept as capital at the end of the previous period, then the producible goods $y^{P} \geqq 0$ can be produced in the present period by using the primary goods $y^{0} \leqq 0$. Note that $y^{P}+y^{0}=y$.

(5) Consumers. There are $I$ consumers each of whom lives over the time horizon. Let $\bar{I}=\{1, \ldots, I\}$. In each period $t=1, \ldots$, consumer $i$ has a consumption set $X^{i}$, a subset of $R^{J}$. $x \in X^{i}$ means that he can supply primary goods $x^{0} \leqq 0$ and consume $x^{P} \geqq 0$. At initial period 0 , he has the initial stock $\kappa_{0}^{i} \in R_{+}^{P}$. He has a concave utility function $u^{i}: X^{i} \rightarrow R$.

(6) Production path and consumption path. A production path $(-k, y)$ is a pair of sequences in $R^{J} \times R^{J}$ such that $k=\left\{k_{0}, k_{1}, \ldots\right\} y=\left\{y_{1}, y_{2}, \ldots\right\}$ and $\left(-k_{t-1}, y_{t}\right) \in Y$ for all $t=1,2, \ldots$. A consumption path for consumer $i, x$, is a sequence in $R^{J}$ such that $\underset{x}{x}=\left\{x_{1}, x_{2}, \ldots\right\}, x_{t} \in X^{i}$ for $t \geqq 1$.

(7) Feasible allocation and feasible path. The word 'allocation' often refers to a single period allocation of commodities, while an allocation of commodities over the time horizon is called an allocation path. A bundle $e=((-k, y)$, $\left.\underline{x}^{1}, \ldots, x^{I}\right)$ is called an allocation path if $(-k, y)$ is a production path and $\tilde{x}^{i}$ is a consumption path of consumer $i$ for $i=1, \ldots, I$. An allocation path, $e$, is feasible from an initial stock $k$, if $y_{t}-k_{t}=\sum_{i=1}^{I} x_{t}^{i}, t \geqq 1$, and if $k_{0}=k$ [Malinvaud (1953)]. 
(8) Adequacy. An initial allocation of capital, $\left(\kappa_{0}^{1}, \ldots, \kappa_{0}^{I}\right) \in R_{+}^{P} \times \cdots \times R_{+}^{P}$, is said to be adequate [see Bewley $(1972,1982)]$ if it satisfies the following:

(a) There are $\varepsilon>0, j \in \bar{C}^{i}$, and $\bar{x}^{i} \in X^{i}, i=1, \ldots, I$, such that $\bar{x}^{i}+\varepsilon \chi^{(j)}=\bar{y}^{i}-\kappa_{0}^{i}$ for some $\left(-\kappa_{0}^{i}, \bar{y}^{i}\right) \in Y$.

(b) There are $\varepsilon$ and $\bar{x} \in X$ such that $\bar{x}+e \chi^{\bar{J}}=\bar{y}-\kappa_{0}$ for some $\left(-\kappa_{0}, \bar{y}\right) \in Y$ where $\kappa_{0}=\sum_{i-1}^{I} \kappa_{0}^{i}$.

(a) means that each consumer can reproduce his own initial stocks with some surplus of a desired good. (b) means that the society can reproduce its own initial stocks with some surplus of every good. Similar assumptions are commonly used in equilibrium theory [see McKenzie (1959), Debreu (1959), and Bewley $(1972,1982)]$ in order to ensure that everybody has positive income. (b) implies expansibility of initial stocks, which is commonly assumed in capital theory [see Gale (1967), Brock (1970), Scheinkman (1976), and McKenzie (1979)].

(9) Discounted utility sum. Each consumer discounts future utilities by a discount factor $\rho$. $\sum_{t=1}^{\infty} \rho^{t} u^{i}\left(x_{t}\right)$ is consumer $i$ 's objective function. ${ }^{\perp}$ Since, by Assumptions (A.6) and (A.8) below, we may normalize utility functions so that $u^{i}(x) \geqq 0$ for all $x \in X^{i}, i=1, \ldots, I$, this infinite sum is well defined.

(10) Price, budget and demand. A price path $p=\left\{p_{0}, p_{1}, \ldots\right\}$ is a sequence of present value prices in $R_{+}^{J}$. Consumer $i$ 's total net spending cannot exceed the value of his initial stock,

$$
\sum_{t=1}^{\infty} p_{t} x_{t}=p_{0} \kappa_{0}^{i} \quad \text { and } \quad x_{t} \in X^{i}, \quad t \geqq 1
$$

(11) Competitive equilibrium path. Given an initial allocation of capital,

$$
\left(\kappa_{0}^{1}, \ldots, \kappa_{0}^{I}\right),\left(e(\rho), \underset{\sim}{p(\rho))}=\left((-\underline{k}(\rho), \underset{\sim}{y}(\rho)), \underline{x}^{1}(\rho), \ldots, \underline{x}^{I}(\rho), \underset{\sim}{p(\rho))}\right.\right.
$$

is a competitive equilibrium path with the discount factor $\rho$ if it satisfies:

(i) $\sum_{t=0}^{\infty}\left|p_{t}(\rho)\right|<\infty$ and $p_{t}(\rho)>0$ for all $t$.

(ii) Utility maximization. ${\underset{\sim}{x}}^{i}(\rho)$ maximizes $\sum_{t=1}^{\infty} \rho^{t} u^{i}\left(x_{t}\right)$ subject to

$$
\begin{aligned}
& \sum_{t=1}^{\infty} p_{t}(\rho) x_{t} \leqq p_{0}(\rho) \kappa_{0}^{i} \text { and } x_{t} \in X^{i}, t \geqq 1, \quad \text { where } \\
& \sum_{t=1}^{\infty} p_{t}(\rho) x_{t}^{i}(\rho)=p_{0}(\rho) \kappa_{0}^{i} .
\end{aligned}
$$

\footnotetext{
${ }^{1}$ All consumers have the same discount factor. This is justified by the fact that less patient consumers eventually consume nothing in the sufficiently far future [Ramsey (1928), Rader (1971), Becker (1980), and Bewley (1982)].
} 
(iii) Profit maximization.

$$
\begin{aligned}
0 & =\sum_{t=1}^{\infty}\left(p_{t}(\rho) y_{t}(\rho)-p_{t-1}(\rho) k_{t-1}(\rho)\right), \\
& \geqq \sum_{t=1}^{\infty}\left(p_{t}(\rho) y_{t}-p_{t-1}(\rho) k_{t-1}\right)
\end{aligned}
$$

for all $\left(-k_{t-1}, y_{t}\right) \in Y, t \geqq 1$, and $\left(-k_{t-1}(\rho), y_{t}(\rho)\right) \in Y, t \geqq 1$.

(iv) Market clearing.

$$
y_{t}(\rho)-k_{t}(\rho)=\sum_{i=1}^{I} x_{t}^{i}(\rho), t \geqq 1 \quad \text { and } \quad k_{0}(\rho)=\sum_{i=1}^{I} \kappa_{0}^{i}
$$

(12) Marginal utility of income. Since cach consumer maximizcs his utility sum under his budget constraint, by the Kuhn-Tucker-Uzawa theorem [Takayama (1974, p. 48)] we may find the set of his marginal utilities of income at the equilibrium path as follows:

$$
\begin{aligned}
\Gamma^{i}(e(\rho), \underline{\sim}(\rho))=\left\{\gamma^{i}\right. & \geqq 0: \sum_{t=1}^{\infty} \rho^{t} u^{i}\left(x_{t}^{i}(\rho)\right)-\gamma^{i} \sum_{t=1}^{\infty} p_{t}(\rho) x_{t}^{i}(\rho) \\
& \left.\geqq \sum_{t=1}^{\infty} \rho^{t} u^{i}\left(x_{t}\right)-\gamma^{i} \sum_{t=1}^{\infty} p_{t}(\rho) x_{t} \text { for all } x_{t} \in X^{i}, t \geqq 1\right\} .
\end{aligned}
$$

We call an element of $\Gamma^{i}(e(\rho), p(\rho))$ consumer $i$ 's marginal utility of income at $(e(\rho), \underset{\sim}{p}(\rho)) . \gamma(\rho)=\left(\gamma^{1}(\rho), \ldots, \gamma^{I}(\tilde{\rho})\right)$ with $\gamma^{i}(\rho) \in \Gamma^{i}(e(\rho), \underset{p}{p}(\rho))$ for all $i$ is called a marginal-utility-of-income vector of the equilibrium path, $(e(\rho), \underset{\sim}{p}(\rho))$; they are expressed as $(e(\rho), \underline{p}(\rho): \gamma(\rho))$.

(13) Social welfare function. A social welfare function is defined as

$$
W(x, \gamma)=\max \left\{\sum_{i=1}^{I} u^{i}\left(x^{i}\right) / \gamma^{i}: \sum_{i=1}^{I} x^{i}=x \text { and } x^{i} \in X^{i} \text { for all } i\right\} .
$$

Under our assumptions, we may prove that $W(x, \gamma)$ is continuous in $x$ and $\gamma$.

(14) Long-run equilibrium. A pair of an allocation and a current price, $\left(e^{\rho}, q^{\rho}\right)=\left(\left(-k^{\rho}, y^{\rho}\right), \quad x^{1 \rho}, \ldots, x^{I \rho}, q^{\rho}\right) \in Y \times X^{1} \times \cdots \times X^{I} \times R_{+}^{J} \quad$ is a long run

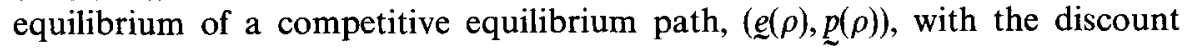
factor $\rho$, if it satisfies:

(i) $q^{\rho}>0$. 
(ii) $x^{\rho}$ maximizes $W(x, \gamma(\rho))$ subject to $q^{\rho} x \leqq\left(\rho^{-1}-1\right) q^{\rho} k^{\rho}$ and $x \in X$. where $x^{\rho}=\sum_{i=1}^{I} x^{i \rho}$ and $X=\sum_{i=1}^{I} X^{i}$.

(iii) $0=q^{\rho}\left(y^{\rho}-\rho^{-1} k^{\rho}\right) \geqq q^{\rho}\left(y-\rho^{-1} k\right)$ for all $(-k, y) \in Y$.

(iv) $y^{\rho}-k^{\rho}=x^{\rho}$.

(v) $W\left(x^{\rho}, \gamma(\rho)\right)=\sum_{i=1}^{I} u^{i}\left(x^{i \rho}\right) / \gamma^{i}(\rho)$.

(vi) $\gamma^{i}(\rho) \in \Gamma^{i}(e(\rho), \underline{p}(\rho)) .^{2}$

Note that a long-run equilibrium $\left(e^{\rho}, q^{\rho}\right)$ depends upon initial allocations of capital of $(e(\rho), \underset{\sim}{p}(\rho))$ since $\gamma(\rho)$ depends upon $(\underset{\sim}{e}(\rho), \underset{\sim}{p}(\rho))$.

(15) Normalization of prices. For an equilibrium path and its marginalutility-of-income vector $(e(\rho), p(\rho): \gamma(\rho))$, we normalize the equilibrium price path $p(\rho)$ so that $\sum_{i=1}^{I} \gamma^{i}(\rho)=1$. By the Kuhn-Tucker-Uzawa theorem, the social welfare maximization condition, (14.ii), implies that there is $\alpha>0$ such that $W\left(x^{\rho}, \gamma(\rho)\right)-\alpha q^{\rho} x^{\rho} \geqq W(x, \gamma(\rho))-\alpha q^{\rho} x$ for all $x \in X$. We normalize $q^{\rho}$ so that $\alpha=1$. Then,

$$
W\left(x^{\rho}, \gamma(\rho)\right)-q^{\rho} x^{\rho} \geqq W(x, \gamma(\rho))-q^{\rho} x \quad \text { for all } x \in X .
$$

(16) Equilibrium pair. $\pi^{\rho}=\left(e(\rho), p(\rho), e^{\rho}, q^{\rho}: \gamma(\rho)\right)$ is called an equilibrium pair with an initial allocation of capital if $(e(\rho), p(\rho))$ is a competitive equilibrium path from the initial allocation, if $\gamma(\rho) \in \Gamma(\tilde{e}(\rho), \underline{p}(\rho))$, and if $\left(e^{\rho}, q^{\rho}\right)$ is a longrun equilibrium of $(e(\rho), p(\rho))$.

(17) Ramsey point and a limit pair. A pair of an allocation and a price with a marginal-utility-of-income vector, $\left(e^{*}, q^{*}: \gamma(\rho)\right)$, is a Ramscy point associated with a given initial allocation of capital if there is a sequence of equilibrium pairs, $\left.\left\{\pi^{\rho_{n}}\right\}=\left\{e\left(\rho_{n}\right), p\left(\rho_{n}\right), e^{\rho_{n}}, q^{\rho_{n}}: \gamma\left(\rho_{n}\right)\right)\right\}$, with $\rho_{n} \rightarrow 1$ such that $\left(e\left(\rho_{n}\right), p\left(\rho_{n}\right)\right)$ is an equilibrium path from the initial allocation for all $\tilde{n}$ and that $\left(e^{\rho_{n}}, q^{\rho_{n}}: \gamma\left(\rho_{n}\right)\right)$ converges to $\left(e^{*}, q^{*}: \gamma^{*}\right)$. Moreover, if $\left(e\left(\rho_{n}\right), \underline{p}\left(\rho_{n}\right)\right)$ also converges to $(e(1), p(1))$ pointwise, we say that $\pi^{\rho_{n}}$ converges to a limit pair $\pi^{1}=\left(e(1), p(1), e^{*}, q^{*}: \gamma^{*}\right) \cdot e^{*}\left(q^{*}\right)$ is called a Ramsey allocation (price). $(e(1), p(1))$ is called a pseudo-equilibrium path, since a competitive equilibrium path is defined for the case where the future is discounted.

(18) McKenzie equilibrium. Define the stationary net production set $Z^{1}$ as follows: $Z^{1}=\left\{z \in R^{J}: z=y-k,(-k, y) \in Y\right\}$. A pair of an allocation and a price, $\left(e^{*}, q^{*}\right)=\left(\left(-k^{*}, y^{*}\right), x^{1 *}, \ldots, x^{I *}, q^{*}\right)$, is called a McKenzie equilibrium if it satisfies:

(i) $q^{*}>0$.

\footnotetext{
${ }^{2}$ This characterization is an extension of that of a quasi-stationary optimal path in capital theory [see Sutherland (1970), Peleg and Ryder (1974), McKenzie (1979), and Bewley (1982)].
} 
(ii) $x^{i *}$ maximizes $u^{i}(x)$ subject to $q^{*} x \leqq 0$.

(iii) $0=q^{*} z^{*} \geqq q^{*} z$ for all $Z^{1}$ where $z^{*}=y^{*}-k^{*} \in Z^{1}$.

(iv) $z^{*}=\sum_{i=1}^{I} x^{i *}$.

We call $\left(e^{*}, q^{*}\right)$ a McKenzie equilibrium since this type of general equilibrium model is studied by McKenzie $(1959,1981)$. We will show that if the McKenzie equilibrium is uniquely determined, it is the turnpikc. It characterizes the following stationary state. The present prices of future goods, $q^{*}$, are equal to those of present goods. Each consumer repeats activity $x^{i *}$, $i=1, \ldots, I$, maximizing his utility in each period by spending as much as he earns $q^{*} x^{i *}=0$. Producers choose capital input in a period and output and primary-good input in the next period facing price $q^{*}$ in both periods; $q^{*} y^{*}-q^{*} k^{*}=0$ since $Y$ is a cone. The demand meets the supply every period; $y^{*}-k^{*}=\sum_{i=1}^{I} x^{i *}$.

(19) Non-myopic Pareto efficiency. It is said that consumer $i$ strongly (weakly) prefers a consumption path $x^{\prime}$ to another path $\underset{x}{ }$ with respect to a non-myopic preference if $\lim _{\sup _{T \rightarrow \infty}} \tilde{\sum}_{t=1}^{T}\left(u^{i}\left(x_{t}\right)-u^{i}\left(x_{t}^{\prime}\right)\right)<0(\leqq 0) .{ }^{3}$ A feasible allocation path $e=\left((-\underline{w}, y), \underline{x}^{1}, \ldots, x^{I}\right)$ from $k$ is said to be Pareto efficient with respect to non-myopic preferences if it satisfies the following. If for an allocation path $\underline{e}^{\prime}=\left(\left(-\underline{k}^{\prime}, \underline{y}^{\prime}\right), \underline{x}^{\prime 1}, \ldots, \underline{x}^{\prime I}\right), \underline{x}^{i}$ is weakly preferred to $x^{i}$ with respect to a non-myopic preference for all $i$ and strongly preferred for at least one $i$, then ${\underset{e}{e}}^{\prime}$ is not fcasible from $k$. A fcasible allocation path $\underset{e}{e}$ from $k$ is said to be $\varepsilon$-almost Pareto efficient with respect to non-myopic preferences if there is a feasible allocation path $\underline{e}^{\prime}$ from $k$ such that $\underline{e}^{\prime}$ is Pareto efficient with respect to non-myopic preferences and that $\left|e_{t}-e_{t}^{\prime}\right| \dot{<} \varepsilon$ for all $t \geqq 0$.

\section{Assumptions and results}

\subsection{Assumptions}

Assumptions (A.1)-(A.18) below are assumed in Yano (1984) in order to prove the Liapounov stability for a long run equilibrium (see Proposition 1 in section 4). They are weaker than the assumptions made by Bewley (1982) in a few respects. Readers are referred to Yano (1984) for detailed discussions on the assumptions. We assume

(A.1). $\quad \bar{P} \neq \phi, \overline{0} \neq \phi$, and $\bar{P} \cup \overline{0}=\bar{J}$.

(A.2). $\quad Y$ is a closed convex cone in $R^{P} \times R^{J}$ with the vertex at zero.

(A.3). $\quad(-k, y) \in Y$ implies $k \geqq 0$ and $y^{0} \leqq 0$.

\footnotetext{
${ }^{3}$ In the literature of capital theory, $x^{\prime}$ is said to catch up to $x$ if $x^{\prime}$ is weakly preferred to $\underset{x}{ }$ with respect to a non-myopic preference [see von Weizsäcker (1965), Atsumi (1965), Gale (1967) and Brock (1970)].
} 
(A.4). Necessity of primary goods. $(-k, y) \in Y$ and $y^{P}>0$ imply that $y^{0}<0$.

(A.5). Free disposal. $(-k, y) \in Y$ and $\left(-k^{\prime}, y^{\prime}\right) \leqq(-k, y)$ with $k^{\prime} \in R_{+}^{P}$ imply that $\left(-k^{\prime}, y^{\prime}\right) \in Y$.

(A.6). There is $a^{i} \in R^{C^{i}}, i=1, \ldots, I$, such that $X^{i}=a^{i}+R^{C^{i}}, a^{i P} \geqq 0$ and $a^{i 0} \leqq 0{ }^{9}$

(A.7). $\quad u^{i}: X^{i} \rightarrow R$ is concave and continuous.

(A.8). $\quad \bar{C}^{i} \neq \phi, i=1, \ldots, I$. For any $\alpha>0, u^{i}\left(x+\alpha \chi^{\{j\}}\right)>u^{i}(x)$ if $j \in \bar{C}^{i}$.

(A.9). $\bar{C} \cap \bar{P} \neq \phi$ where $\bar{C}=U_{i=1}^{I} \bar{C}^{i}$.

(A.10). All consumers have a common discount factor $0<\rho<1$.

(A.11). Non-triviality of long run equilibrium allocation. There are $\bar{\varepsilon}>0$ and $\hat{\rho}>0$ such that for any long-run equilibrium allocation with $\hat{\rho} \leqq \rho<1, e^{\rho}=$ $\left(\left(-k^{\rho}, y^{\rho}\right), x^{1 \rho}, \ldots, x^{I \rho}\right)$, it holds that $\left|x^{\rho P}\right| \geqq \bar{\varepsilon}$ where $x^{\rho}=\sum_{i=1}^{I} x^{i \rho}$.

(A.12). Sufficiency of long-run equilibrium consumption. For any long-run equilibrium with $\hat{\rho} \leqq \rho<1$, there is $0<\alpha<1$ such that $\alpha \leqq \alpha<1$ implies $\alpha x^{i \rho P}+x^{i \rho 0} \in X^{i}, i=1, \ldots, I$.

(A.13). Substitutability. Let $\left(-k, k^{\prime}+x\right) \in Y, x \in X$ and $k^{\prime} \in R_{+}^{P}$. For any $\varepsilon^{\prime}>0$, there is $\varepsilon>0$ such that $\left|x^{P}\right| \geqq \varepsilon^{\prime}$ implies $\left(-k, k^{\prime}+\varepsilon \chi^{P}+x^{0}\right) \in Y$.

(A.14). Uniformly bounded marginal productivity of capital. Let $(-k, y)$ and $\left(-k^{\prime}, y^{\prime}\right)$ be on the boundary of $Y$. If for some long-run equilibrium capital stock, $k^{\rho}$, with $\hat{\rho} \leqq \rho<1,\left|k-k^{\rho}\right|<\bar{\varepsilon}$ and $\left|k^{\prime}-k^{\rho}\right|<\bar{\varepsilon}$, and if $k^{\prime} \geqq k, y^{\prime P} \geqq y^{P}$ and $y^{\prime 0}=y^{0}$, then there is $\eta>0$ such that $\left|y^{\prime P}-y^{P}\right| \leqq \eta\left|k^{\prime}-k\right|$.

(A.15). Around the set of all Ramsey points, $Y$ is a strictly convex cone and $u^{i}$ is strictly concave. ${ }^{4}$

Under Assumptions (A.1)-(A.15) we may prove that there is $0<\bar{\rho}<1$ such that for any adequate initial allocation of capital and for any $\rho$ such that $\bar{\rho} \leqq \rho<1$, there is an equilibrium pair [see Yano (1984)].

In addition to these assumptions we assume

(a.l). The production set $Y$ can be expressed as a continuous production function $f: R^{P} \times R^{J} \rightarrow R$ such that $f(-k, y) \leqq 0$ if and only if $(-k, y) \in Y$.

\footnotetext{
${ }^{4} Y$ is said to be a strictly convex cone around the set of all Ramsey points if and only if there is $\bar{\varepsilon}>0$ such that, for any Ramsey allocation $\left(\left(-k^{*}, y^{*}\right), x^{1 *}, \ldots, x^{i *}\right),\left|\left(-k^{\prime}, y^{\prime}\right)-\left(-k^{*}, y^{*}\right)\right|<\bar{\varepsilon}$, $\left|\left(-k^{\prime \prime}, y^{\prime \prime}\right)-\left(-k^{*}, y^{*}\right)\right|<\bar{\varepsilon},\left(-k^{\prime}, y^{\prime}\right) \in Y,\left(-k^{\prime \prime}, y^{\prime \prime}\right) \in Y$, and $\left(-k^{\prime}, y^{\prime}\right) \neq \beta\left(-k^{\prime \prime}, y^{\prime \prime}\right)$ for any $\beta \geqq 0$ imply that $\alpha\left(-k^{\prime}, y^{\prime}\right)+(1-\alpha)\left(-k^{\prime \prime}, y^{\prime \prime}\right) \in$ interior $Y, 0<\alpha<1$. Also $u^{i}$ is said to be strictly concave around the set of all Ramsey points if and only if there is $\bar{\varepsilon}>0$ such that, for any Ramsey allocation $\left(\left(-k^{*}, y^{*}\right), x^{1 *}, \ldots, x^{1 *}\right),\left|x^{\prime}-x^{i *}\right|<\bar{\varepsilon},\left|x^{\prime \prime}-x^{i *}\right|<\bar{\varepsilon}, x^{\prime} \in X^{i}, x^{\prime \prime} \in X^{i}$, and $x^{\prime}+x^{\prime \prime}$ imply that $u^{i}\left(\alpha x^{\prime}+\right.$ $\left.(1-\alpha) x^{\prime \prime}\right)>\alpha u^{i}\left(x^{\prime}\right)+(1+\alpha) u^{i}\left(x^{\prime \prime}\right), 0<\alpha<1$.
} 
Moreover, $f$ has continuous first partial derivatives at the production vector $\left(-k^{*}, y^{*}\right)$ of Ramsey allocation $\left(\left(-k^{*}, y^{*}\right), x^{1 *}, \ldots, x^{I *}\right)$.

(a.2). There is at least one $i \subset \bar{I}$ such that for any Ramsey allocation $\left(\left(-k^{*}, y^{*}\right), x^{1 *}, \ldots, x^{I *}\right)$, the consumption vector $x^{i *}$ lies in the interior of $X^{i}$ and $u^{i}$ has continuous first partial derivatives at $x^{i *}$.

Assumption (a.1) implies that the boundary of $Y$ is smooth around the production vectors of Ramsey allocations. Assumption (a.2) is implied by the more basic assumption that there is a consumer whose indifference curves do not intersect the boundary of his consumption set and are smooth.

\subsection{Results}

We will prove the following theorems:

\section{Theorem 1. A Ramsey point is a McKenzie equilibrium}

Theorem 2. Turnpike theorem. Suppose that the single-period general equilibrium model (18.i)-(18.iv) has the unique McKenzie equilibrium, $\left(e^{*}, q^{*}\right)$. Then $\left(e^{*}, q^{*}\right)$ is independent of initial allocations of capital. Moreover, for any adequate initial allocation of capital and for any $\varepsilon>0$ there is $0<\rho^{\prime}<1$ such that the following holds. There is $T \geqq 1$ such that if $(e(\rho), p(\rho))$ is an equilibrium path from the initial allocation and if $\rho^{\prime} \leqq \rho<1,\left|e_{t}(\rho)-e^{*}\right|<\varepsilon$ and $\left|q_{t}(\rho)-q^{*}\right|<\varepsilon$ for all $t \geqq T$, where $q_{t}(\rho)=\rho^{-t} p_{t}(\rho)$.

Theorem 2 implies that the allocation path $\left(e^{*}, q^{*}\right)=\left\{\left(e_{t}^{*}, q_{t}^{*}\right)\right\}$ such that $\left(e_{t}^{*}, q_{t}^{*}\right)=\left(e^{*}, q^{*}\right)$ for all $t$ is the turnpike of our economy. The theorem does not imply that $\left(e_{t}(\rho), q_{t}(\rho)\right)$ converges to $\left(e^{*}, q^{*}\right)$, but implies only that it converges to a small neighborhood of $\left(e^{*}, q^{*}\right)$. This type of a turnpike theorem is called a neighborhood turnpike theorem [McKenzie (1979)].

Theorem 3. For any equilibrium allocation path e( $\rho$ ) from a given adequate initial allocation of capital and for any $\varepsilon>0$, there is $0<\rho^{\prime}<1$ such that the following holds: $\rho^{\prime} \leqq \rho<1$ implies that $\mathrm{e}(\rho)$ is $\varepsilon$-almost Pareto efficient with respect to non-myopic preferences.

Corollary 1. The allocation path, $\mathfrak{e}^{*}$, of the turnpike is Pareto efficient with respect to non-myopic preferences.

\section{Proofs of theorems}

Here, we will prove the theorems above. Let $\bar{\rho}$ be as in section 3. For each pair of an adequate initial allocation of capital and a discount factor $\rho$ such 
that $\bar{\rho} \leqq \rho<1$, there may be multiple equilibrium. Take an adequate initial allocation, $\left(\kappa_{0}^{1}, \ldots, \kappa_{0}^{I}\right)$. For this initial allocation and for each discount factor $\rho$ such that $\bar{\rho} \leqq \rho<1$, we select an equilibrium pair and denote it as $\pi^{\rho}=\left(\underset{\sim}{e}(\rho), p(\rho), e^{\rho}, q^{\rho}, q^{\rho}: \gamma(\rho)\right), \underset{\sim}{e}(\rho)=\left\{e_{t}(\rho)\right\}, e_{t}(\rho)=\left(\left(-k_{t-1}(\rho), y_{t}(\rho)\right)\right.$, $\left.x_{t}^{1}(\rho), \ldots, x_{t}^{I}(\rho)\right), \tilde{p}(\rho)=\left\{p_{t}(\rho)\right\}$, and $e^{\rho}=\left(\left(-k^{\rho}, y^{\rho}\right), x^{1 \rho}, \ldots, x^{I \rho}\right)$. Moreover, let $q_{t}(\rho)=\rho^{-t} p_{t}(\rho)$. We will first prove Theorem 1 .

Proof of Theorem 1. Take a Ramsey point $\left(e^{*}, q^{*}: \gamma(1)\right)$ to which a sequence $\left(e^{\rho_{n}}, q^{\rho_{n}}: \gamma\left(\rho_{n}\right)\right)$ converges. In the next section, we will prove:

Lemma 1. There are $\beta>0, \beta^{\prime}>0$, and $0<\rho^{\prime}<1$ such that $\rho^{\prime} \leqq \rho<1$ implies the following. $\left|e_{t}(\rho)\right|<\beta$ for all $t \geqq 1,\left|q_{t}(\rho)\right|<\beta$ for all $t,\left|e^{\rho}\right|<\beta, \beta^{\prime}<\left|q^{\rho}\right|<\beta$, and $\gamma^{i}(\rho) \geqq \beta^{\prime}$ for all $i$.

Lemma 1 implies that there is a subsequence of $\rho_{n}$, call it $\rho_{n}$ again, such that $\pi^{\rho_{n}}$ converges to $\pi^{1}=\left(e(1), p(1), e^{*}, q^{*}: \gamma(1)\right)$. Denote $e(1)=\left\{e_{t}(1)\right\}$, $e_{t}(1)=\left(\left(-k_{t-1}(1), y_{t}(1)\right) . \quad x_{t}^{1}(1), \ldots, x_{t}^{I}(1)\right), p(1)=\left\{p_{t}(1)\right\}$, and $e^{*}=\left(\left(-\mathrm{k}^{*}, y^{*}\right)\right.$, $\left.x^{1 *}, \ldots, x^{I *}\right)$.

Since $q^{\rho_{n}} \rightarrow q^{*} \neq 0$ by Lemma $1,(18 . i)$ holds by (14.i). Since $q^{\rho_{n}} \rightarrow q^{*} \neq 0$ and $\left(-k^{\rho_{n}}, y^{\rho_{n}}\right) \rightarrow\left(-k^{*}, y^{*}\right)$, (14.iii) implies (18.iii) and (14.iv) implies (18.iv). Note that (15.i) implies $u^{i}\left(x^{i \rho}\right)-\gamma^{i}(\rho) q^{\rho} x^{i p} \geqq u^{i}(x)-\gamma^{i}(\rho) q^{\rho} x$ for all $x \in X^{i}$, for all $i$. Thus, since $x^{i \rho_{n}} \rightarrow x^{i *}, \quad q^{\rho_{n}} \rightarrow q^{*} \neq 0$, and $\gamma^{i}\left(\rho_{n}\right) \rightarrow \gamma^{i}(1)$, we have $u^{i}\left(x^{i *}\right)$ $-\gamma^{i}(1) q^{*} x^{i *} \geqq u^{i}(x)-\gamma^{i}(1) q^{*} x$, for all $x \in X^{i}$. Thus, if we prove $q^{*} x^{i *}=0$ for all $i$, (18.ii) holds. Therefore, in order to prove the theorem, it suffices to show $q^{*} x^{i *}=0$ for all $i$.

We will use the following lemma (see the next section for a proof).

Lemma 2. For any $\varepsilon>0$ there are $0<\rho^{\prime}<1$ and $T \geqq 1$ such that $\rho^{\prime} \leqq \rho \leqq 1$ implies $\left|e_{t}(\rho)-e^{\rho}\right|<\varepsilon$ and $\left|q_{t}(\rho)-q^{\rho}\right|<\varepsilon$ for all $t \geqq T$.

Define $\bar{I}_{+}=\left\{i \in \bar{I}: q^{*} x^{i *}>0\right\}$ and $\bar{I}_{-}=\left\{i \in \bar{I}: q^{*} x^{i *}<0\right\}$. By the definition of $I_{+}$we may choose $\varepsilon>0$ and $\delta>0$ such that $\left|q-q^{*}\right|<\delta$ and $\left|x-x^{i *}\right|<\delta$ imply $q x \geqq \varepsilon / 2$ for all $i \in \bar{I}_{+}$. Since $\left(e^{\rho^{n}}, q^{\rho^{n}}\right) \rightarrow\left(e^{*}, q^{*}\right)$, there is $N^{\prime}$ such that $\mid q^{\rho^{n}}-$ $q^{*} \mid<\delta / 2$ and $\left|x^{i \rho^{n}}-x^{i *}\right|<\delta / 2$ for all $n \geqq N^{\prime}$ and all $i \in \bar{I}$. Since $\rho_{n} \rightarrow 1$, by Lemma 2 there is $N \geqq N^{\prime}$ and $T$ such that $\left|q_{t}\left(\rho^{n}\right)-q^{\rho^{n}}\right|<\delta / 2$ and $\mid x_{t}^{i}\left(\rho^{n}\right)-$ $x^{i \rho^{n}} \mid<\delta / 2$ for all $n \geqq N$ and all $t \geqq T$. Thus, since, for all $n \geqq N$ and $t \geqq T\left|q_{t}\left(\rho^{n}\right)-q^{*}\right|<\delta$ and $\left|x_{t}^{i}\left(\rho^{n}\right)-x^{i *}\right|<\delta$, the definition of $\delta$ implies $q_{t}\left(\rho^{n}\right) x_{t}^{i}\left(\rho^{n}\right) \geqq \varepsilon / 2$ for all $i \in \bar{I}_{+}$. Therefore, by using a similar method for $i \in \bar{I}_{-}$, we may establish that there are $N$ and $T$ such that $i \in \bar{I}_{+}\left(i \in \bar{I}_{-}\right)$implies $q_{t}\left(\rho^{n}\right) x_{t}^{i}\left(\rho^{n}\right)>0(<0)$ for all $n \geqq N$ and $t \geqq T$.

In order to show the theorem, it suffices to show that $\bar{I}_{1}=\bar{I}=\emptyset$. Suppose $\bar{I}_{+} \neq \emptyset$. Let $i \in \bar{I}_{+}$and $T$ and $N$ be as above. Lemma 1 implies that 
$\left|p_{t}\left(\rho^{n}\right)\right|$ and $\left|x_{t}^{i}\left(\rho^{n}\right)\right|$ are uniformly bounded with respect to $t$ and $n$. Thus, consumer $i$ 's budget equation implies that there is $\beta>0$ such that

$$
\sum_{t=T}^{\infty} p_{t}\left(\rho^{n}\right) x_{t}^{i}\left(\rho^{n}\right)=p_{0}\left(\rho^{n}\right) \kappa_{0}^{i}-\sum_{t=1}^{T-1} p_{t}\left(\rho^{n}\right) x_{t}^{i}\left(\rho^{n}\right) \leqq \beta,
$$

for all $n \geqq N$. Since $p_{t}\left(\rho^{n}\right) x_{t}^{i}\left(\rho^{n}\right) \geqq 0$ for all $n \geqq N$ and $t \geqq T$ by the choice of $N$ and $T$, Fatou's lemma [Rudin (1976)] implies

(ii) $\quad \sum_{t=1}^{\infty} \lim _{n \rightarrow \infty} p_{t}\left(\rho^{n}\right) x_{t}^{i}\left(\rho^{n}\right) \leqq \lim _{n \rightarrow \infty} \sum_{t=T}^{\infty} p_{t}\left(\rho^{n}\right) x_{t}^{i}\left(\rho^{n}\right)$.

Since $p_{t}\left(\rho^{n}\right) x_{t}^{i}\left(\rho^{n}\right) \rightarrow p_{t}(1) x_{t}^{i}(1) \geqq 0$, eqs. (i) and (ii) imply

$$
0 \leqq \sum_{t=T}^{\infty} p_{t}(1) x_{t}^{i}(1) \leqq \beta .
$$

Since $p_{t}(1) x_{t}^{i}(1) \geqq 0$ for all $t \geqq T$, inequality (iii) implies

$$
p_{t}(1) x_{t}^{i}(1) \rightarrow 0
$$

as $t \rightarrow \infty$. In the next section, we will prove

Lemma 3. $e_{t}(1) \rightarrow e^{*}$ and $p_{t}(1) \rightarrow q^{*}$ as $t \rightarrow \infty$.

By (iv) and Lemma 3, $p_{t}(1) x_{t}^{i}(1) \rightarrow q^{*} x^{i *}=0$. This contradicts that $i \in \bar{I}_{+}$. Thus, $\bar{I}_{+}=\phi$. Since $\bar{I}_{-}=\phi$ can be shown in the same way, Theorem 1 is proved.

We now prove Theorem 2 .

Proof of Theorem 2. By Theorem 1 and the hypothesis of Theorem 2 that there is the unique McKenzie equilibrium $\left(e^{*}, q^{*}\right)$, the Ramsey point is also unique. Since the system (18.i)-(18.iv) is independent of initial allocations of capital, so is $\left(e^{*}, q^{*}\right)$. Therefore, by the definition of a Ramsey point, for any $\varepsilon>0$ there is $0<\rho^{\prime \prime}<1$ such that $\rho^{\prime \prime} \leqq \rho<1$ implies $\left|e^{\rho}-e^{*}\right|<\varepsilon / 2$ and $\left|q^{\rho}-q^{*}\right|<\varepsilon / 2$ for any equilibrium pair with any adequate initial allocation of capital.

For equilibrium pairs $\left(e(\rho), p(\rho), e^{\rho}, q^{\rho}: \gamma(\rho)\right)$ with a given adequate initial allocation, choose $\rho^{\prime \prime} \leqq \rho^{\prime}<1$ and $T \geqq 1$ so that $\rho^{\prime} \leqq \rho<1$ and $t \geqq T$ imply $\left|e_{t}(\rho)-e^{\rho}\right|<\varepsilon / 2$ and $\left|q_{t}(\rho)-q^{\rho}\right|<\varepsilon / 2$, where $q_{t}(\rho)=\rho^{-t} p_{t}(\rho)$. Since $\rho^{\prime} \leqq \rho<1$ and $t \geqq 1$ imply that $\left|e_{t}(\rho)-e^{*}\right|<\varepsilon$ and $\left|q_{t}(\rho)-q^{*}\right|<\varepsilon$, Theorem 2 is proved.

We now prove Theorem 3 . 
Proof of Theorem 3. Suppose $e\left(\rho^{n}\right)$ and $e^{\rho^{n}}$ of equilibrium pair $\pi^{\rho^{n}}$ with a given adequate $\left(\kappa_{0}^{1}, \ldots, \kappa_{0}^{I}\right)$ converge to $e(1)$ and $e^{*}$, respectively, of its limit pair, $\pi^{1}$. We first show that $e\left(\rho^{n}\right)$ converges to $e(1)$ uniformly with respect to $t$.

Choose $\varepsilon>0$ arbitrarily. Sine $e^{\rho_{n}} \rightarrow e^{*}$, there is $N>0$ such that $\left|e^{\rho_{n}}-e^{*}\right|<\varepsilon / 3$ for all $n \geqq N$. By Lemmas 2 and 3 above, there are $T>0$ and $N^{\prime} \geqq N$ such that $\left|e_{t}(1)-e^{*}\right|<\varepsilon / 3$ and $\left|e_{t}\left(\rho_{n}\right)-e^{\rho_{n}}\right|<\varepsilon / 3$ for all $t \geqq T$ and $n \geqq N^{\prime}$. Then for all $t \geqq T$ and $n \geqq N^{\prime}$, we have that $\left|e_{t}\left(\rho_{n}\right)-e_{t}(1)\right| \leqq\left|e_{t}(\rho)-e^{\rho_{n}}\right|+\left|e^{\rho_{n}}-e^{*}\right|+$ $\left|e^{*}-e_{t}(1)\right|<\varepsilon$. Since $e_{(}\left(\rho_{n}\right) \rightarrow e(1)$, pointwise, we may choose $N^{\prime \prime} \geqq N^{\prime}$ such that $\left|e_{t}\left(\rho_{n}\right)-e_{t}(1)\right|<\varepsilon$ for all $t \leqq T$ and $n \geqq N^{\prime \prime}$. Thus since we have that $\mid e\left(\rho_{n}\right)-$ $\left.e(1)\right|_{\infty}<\varepsilon$ for all $n \geqq N^{\prime \prime}, e\left(\rho_{n}\right)$ converges to $e(1)$, uniformly.

In order to complete the proof, suppose that $e(1)$ is not Pareto efficient with respect to non-myopic preferences. Then there is a feasible allocation path $e^{\prime}=\left(\left(-\underline{k}^{\prime}, y^{\prime}\right), x^{\prime 1}, \ldots, x^{\prime I}\right)$ from $\sum_{i=1}^{I} k_{0}^{i}$ such that $\lim \sup _{T \rightarrow \infty} \sum_{t=1}^{T}$ $\left(u^{i}\left(x_{t}^{i}(1)\right)-u^{i}\left(x_{t}^{i}\right)\right) \tilde{~} \leqq$ for all $i$ with strict inequality for some $i$, where $x^{i}(1)$ is of $e(1)$. Let $\gamma(1)$ be of limit pair $\pi^{1}$. Then, it holds,

$$
\begin{aligned}
0 & <\sum_{i=1}^{I} \frac{1}{\gamma^{i}(1)} \liminf _{T \rightarrow \infty} \sum_{t=1}^{T}\left(u^{i}\left(x_{t}^{i}\right)-u^{i}\left(x_{t}^{i}(1)\right)\right), \\
& \leqq \liminf _{T \rightarrow \infty} \sum_{t=1}^{T} \sum_{i=1}^{I} \frac{1}{\gamma^{i}(1)}\left(u^{i}\left(x_{t}^{i}\right)-u^{i}\left(x_{t}^{i}(1)\right)\right), \\
& \leqq \limsup _{T \rightarrow \infty} \sum_{t=1}^{T}\left(W\left(x_{t}^{\prime}, \gamma(1)\right)-W\left(x_{t}(1), \gamma(1)\right)\right),
\end{aligned}
$$

where $x_{t}^{\prime}=\sum_{i=1}^{I} x_{t}^{\prime i}$. This contradicts the following lemma (see the next section for a proof).

Lemma 4. Pseudo-equilibrium path e(1) of limit pair $\pi^{1}$ is (overtaking) optimal with respect to $\{W(x, \gamma(1))\}$ where $\gamma(1)$ is of $\pi^{1}$; i.e., for any feasible path e from the initial allocation of $\underset{e}{(1)}, \lim \sup _{T \rightarrow \infty} \sum_{t=1}^{T}\left(W\left(x_{t}, \gamma(1)\right)-W\left(x_{t}(1), \gamma(1)\right)\right) \leqq 0$, where $x_{t}=\sum_{i=1}^{I} x_{t}^{i}, x_{t}(1)=\sum_{i=1}^{I} x_{t}^{i}(1), \underline{x}^{i}$ is of $\underset{\sim}{e}$, and $\underline{\sim}^{i}(1)$ is of $\underset{\sim}{e(1)}$.

This completes the proof of Theorem 3 .

Corollary 1 may be proved in the same way. We therefore omit a proof.

Remark 1. Theorem 3 and Corollary 1 can be established without assuming Assumptions (a.1) and (a.2). These assumptions are used only to establish Lemmas 2 and 3. In the proof of Theorem 3, we may use Sublemmas 7 and 9 in the next section, which do not require (a.1) and (a.2), instead of Lemmas 2 and 3. 
Remark 2. Another preference relation for a consumer who does not discount the future may be considered by using weak maximality [Brock (1970)]. That is, a path $x^{\prime}$ may be said to be strongly (weakly) preferred to another path $\underset{\sim}{x}$ if $\liminf _{T \rightarrow \infty} \sum_{t=1}^{T}\left(u^{i}\left(x_{t}\right) \quad u^{i}\left(x_{t}^{\prime}\right)\right)<0(\leqq 0)$. The definition of Pareto efficiency may be based upon this preference. Unfortunately, however, Pareto efficiency of a pseudo-equilibrium path cannot be shown under this definition; although the first inequality of (i) holds for 'limsup' instead of 'lim inf', the second inequality does not hold in the case of 'lim sup'.

This fact is due to the difference in 'strength' of these two preference orderings. If a sequence $\left\{u_{t}^{\prime}\right\}$ of utilities is strongly preferred to $\left\{u_{t}\right\}$ in the 'lim sup' sense, then there is some $T$ such that $\sum_{\tau=1}^{t} u_{\tau}<\sum_{\tau=1}^{t} u_{\tau}^{\prime}$ for all $t \geqq T$. There may not be such $T$ even if $\left\{u_{t}^{\prime}\right\}$ is strongly preferred to $\left\{u_{t}\right\}$ in the 'lim inf' sense. It merely implies that $\sum_{\tau=1}^{t} u_{\tau}<\sum_{\tau=1}^{t} u_{\tau}^{\prime}$ for infinitely many $t$. Thus, the 'limsup' preference has 'stronger' implications than the 'lim inf' one.

\section{Proof of lemmas}

Lemmas 1 and 4 can be proved without using Assumptions (a.1) and (a.2). Let $\pi^{\rho}$ and the variables associated with $\pi^{\rho}$ be as in the first paragraph of the previous section. We first prove Lemma 1.

To begin with, we may prove the following sublemma [for a proof see Yano (1984, Lemmas 3, 4, 6, and 7)].

Sublemma 1. There are $\beta>0, \beta^{\prime}>0$, and $0<\tilde{\rho}<1$ such that $\tilde{\rho} \leqq \rho<1$ implies the following. $\left|e_{t}(\rho)\right|<\beta$ for all $t \geqq 0,\left|e^{\rho}\right|<\beta, \beta^{\prime}<\left|q^{\rho}\right|<\beta$, and $\gamma^{i}(\rho) \geqq \beta^{\prime}$ for all $i$.

For each $\rho$ such that $\tilde{\rho} \leqq \rho<1$, we define

$$
\Delta_{t}^{\rho}=W\left(x^{\rho}, \gamma(\rho)\right)-q^{\rho} x^{\rho}-\left(W\left(x_{t}(\rho), \gamma(\rho)\right)-q^{\rho} x_{t}(\rho)\right)-q^{\rho}\left(y_{t}(\rho)-\rho^{-1} k_{t-1}(\rho)\right),
$$

where $\tilde{\rho}$ is as in Sublemma 1 and $x_{t}(\rho)=\sum_{i=1}^{I} x_{t}^{i}(\rho)$. By (14.iii) and (15.i), $\Delta_{t}^{\rho} \geqq 0$ for all $\rho$ and $t$. Moreover, define $A_{t}^{\rho}=\sum_{\tau=1}^{\infty} \rho^{\tau} \Delta_{t+\tau}^{\rho}$. Since, by Sublemma $1, \Delta_{t}^{\rho}$ is bounded uniformly in $\rho$ and $t, A_{t}^{\rho}$ is well defined and $A_{t}^{\rho} \geqq 0$ for all $t$ and $\rho$ such that $\tilde{\rho} \leqq \rho<1$. By definition, $\tilde{\rho} \leqq \rho<1$ implies

$$
\Delta_{t}^{\rho}=\rho^{-1} \Lambda_{t-1}^{\rho}-\Lambda_{t}^{\rho},
$$

for all $t$. We may prove the following [see Yano (1984), Lemma 11 for a proof]. proof].

Sublemma 2. $A_{0}^{\rho}$ is bounded uniformly in $\rho$ such that $\tilde{\rho} \leqq \rho<1$, where $\tilde{\rho}$ is as in Sublemma 1.

Moreover, we have 
Sublemma 3. There are $c>0, \varepsilon^{\prime}>0$, and $\rho_{1}$ such that $\tilde{\rho} \leqq \rho_{1}<1$ and that $\rho_{1} \leqq \rho<1$ and $0<\varepsilon<\varepsilon^{\prime}$ imply the following. There are $\hat{x} \in X$ and $\left(-k^{\rho}(\varepsilon), \hat{y}\right) \in Y$ such that $\hat{y}=\hat{x}+k^{\rho}+c \chi^{\bar{P}}$ and $\hat{x}^{0}=x^{\rho 0}$, where $k_{j}^{\rho}(\varepsilon)=\max \left\{0, k_{j}-\varepsilon\right\}$ for all $j$ and $\chi^{\widetilde{P}}$ is as in section 3 .

Proof. This can be proved by a method similar to that which proves Lemma 12 in Yano (1984).

The inequality defining $\Gamma^{i}(\underline{e}(\rho), \underline{p}(\rho))$ in (12) readily implies

$$
\rho^{t} u^{i}\left(x_{t}^{i}(\rho)\right)-\gamma^{i}(\rho) p_{t}(\rho) x_{t}^{i}(\rho) \geqq \rho^{t} u^{i}(x)-\gamma^{i}(\rho) p_{t}(\rho) x,
$$

for all $x \in X$, all $t \geqq 1$, all $i$, all $\rho$ such that $\tilde{\rho} \leqq \rho<1$. Thus, by the definition of the social welfare function in (13) and the normalizations of prices in (15), (iii) implies

$$
\rho^{t} W\left(x_{t}(\rho), \gamma(\rho)\right)-p_{t}(\rho) x_{t}(\rho) \geqq \rho^{t} W(x, \gamma(\rho))-p_{t}(\rho) x,
$$

for all $x \in X$, all $t$, and all $\rho$ such that $\tilde{\rho} \leqq \rho<1$. Moreover, (11.iii) implies

$$
0=p_{t}(\rho) y_{t}(\rho)-p_{t-1}(\rho) k_{t-1}(\rho) \geqq p_{t}(\rho) y-p_{t-1}(\rho) k,
$$

for all $(-k, y) \in Y$, all $t \geqq 1$, and all $\rho$ such that $\tilde{\rho} \leqq \rho<1$. Substituting $x^{\rho}=\sum_{i=1}^{I} x^{i \rho}$ and $\left(-k^{\rho}, y^{\rho}\right)$ for $x$ and $(-k, y)$ in inequalities (iv) and (v), respectively, and adding the two inequalities, we have

$$
\sum_{\tau=t+1}^{\infty} \rho^{\tau}\left(W\left(x_{\tau}(\rho), \gamma(\rho)\right)-W\left(x^{\rho}, \gamma(\rho)\right)\right) \geqq p_{t-1}(\rho)\left(k_{t-1}(\rho)-k^{\prime}\right)
$$

for all $t \geqq 0$ and $\rho$ such that $\tilde{\rho} \leqq \rho<1$.

Define the projection of $Y$ to $R^{J}$ as $Y_{P}$, or $Y_{P}=\left\{y \in R^{J}:(-k, y) \in Y\right.$ for some $k\}$. Then, we may prove

Sublemma 4. $X \cap Y_{P}$ has a non-empty interior.

Proof. This may be proved by the method similar to that used to prove Lemma 5 of Yano (1984).

Note that $q_{t}(\rho)=\rho^{-t} p_{t}(\rho)$. We will prove

Sublemma 5, If $\left|k_{t-1}(\rho)-k^{\rho}\right|<\varepsilon^{\prime}$, there is $\beta>0$ such that $\rho_{1} \leqq \rho<1$ implies $\left|q_{t}(\rho)\right| \leqq \beta$, where $\varepsilon^{\prime}$ and $\rho_{1}$ are as in Sublemma 3 .

Proof. By (vi), for any $t \geqq 1$ and $\rho$ such that $\tilde{\rho} \leqq \rho<1$, we have $q_{t}(\rho)\left(k_{t}(\rho)\right.$ $\left.-k^{\rho}\right) \leqq-\sum_{\tau=1}^{\infty} \rho^{\tau}\left(W\left(x^{\rho}\right)-W\left(x_{t}+\tau(\rho)\right)\right)=q^{\rho}\left(k_{t}(\rho)-k^{\rho}\right)-\Lambda_{t}^{\rho} \leqq q^{\rho}\left(k_{t}(\rho)-k^{\rho}\right)$, where $W(x)=W(x, \gamma(\rho))$, where the equality follows from the definitions of $A_{t}^{\rho}$ and $\Delta_{t}^{\rho}$, and where the last inequality follows since $\Lambda_{t}^{\rho} \geqq 0$. Therefore, by 
Sublemma $1, q_{t}(\rho)\left(k_{t}(\rho)-k^{0}\right)$ is bounded from above uniformly in $t \geqq 0$ and $\rho$ such that $\tilde{\rho} \leqq \rho<1$.

If $\left|k_{t-1}(\rho)-k^{\rho}\right| \leqq \varepsilon^{\prime}$, by Sublemma 3 , there is $c>0$ such that, for any $\rho_{1} \leqq \rho<1$, there are $\left(-k_{t-1}(\rho), y^{\prime}\right) \in Y, x^{\prime} \in X$ and $y^{\prime}=x^{\prime}+k^{\rho}+c \chi^{\bar{p}}$. Then, by (iv) and (v), for any $\rho_{1} \leqq \rho<1$, we have $W\left(x_{t}(\rho)\right)+q_{t}(\rho) k_{t}(\rho) \geqq W\left(x^{\prime}\right)+q_{t}(\rho)$ $\left(k^{\rho}+c \chi^{\bar{P}}\right)$. Thus, since $q_{t}(\rho)\left(k_{t}(\rho)-k^{\rho}-c \chi^{\bar{P}}\right) \geqq W\left(x^{\prime}\right)-W\left(x_{t}(\rho)\right)$, Sublemma 1 implies $q_{t}(\rho)\left(k_{t}(\rho)-k^{\rho}-c \chi^{\bar{P}}\right)$ is uniformly bounded from below.

Thus we have some $\beta^{\prime}>0$ such that for any $\rho_{1} \leqq \rho<1$,

$$
-\beta^{\prime} \leqq q_{t}(\rho)\left(k_{t}(\rho)-k^{\rho}-c \chi^{\bar{P}}\right)<q_{t}(\rho)\left(k_{t}(\rho)-k^{\rho}\right)<\beta^{\prime} .
$$

Suppose that $\left|q_{t}^{P}(\rho)\right|$ is not uniformly bounded for $t$ and $\rho$. Then, by Sublemma 1 we may choose $\left\{\rho_{n}, t_{n}\right\}$ with $\rho_{1} \leqq \rho_{n}<1$ and $t_{n} \geqq 0$ such that $\rho_{n} \rightarrow \rho, k_{t_{n}} \rightarrow k^{\prime} k^{\rho_{n}} \rightarrow k^{\rho},\left|q_{t_{n}}^{P}\left(\rho_{n}\right)\right| \rightarrow \infty$ and $q_{t_{n}}^{P}\left(\rho_{n}\right) /\left|q_{t_{n}}^{P}\left(\rho_{n}\right)\right| \rightarrow \phi$. Note $\phi^{P}>0$. But by (vii) $c \phi^{P} \chi^{P}=0$, a contradiction. Thus $\left|q_{t}^{P^{n}}(\rho)\right|$ is uniformly bounded.

Suppose that $\left|q_{t}(\rho)\right|$ is not uniformly bounded in $\rho$ and $t$. Then, by Sublemma 1 , we may choose $\left\{\rho_{n}, t_{n}\right\}$ with $\rho_{1} \leqq \rho_{n}<1$ and $t_{n} \geqq 0$ such that $\rho_{n} \rightarrow \rho$. $\left(-k_{t_{n}-1}\left(\rho_{n}\right), y_{t_{n}}\left(\rho_{n}\right)\right) \rightarrow\left(-k_{-1}^{\prime}, y^{\prime}\right), k_{t_{n}}\left(\rho_{n}\right) \rightarrow k^{\prime}, x_{t_{n}}\left(\rho_{n}\right) \rightarrow x^{\prime}, q_{t_{n}}\left(\rho_{n}\right) /\left|q_{t_{n}}\left(\rho_{n}\right)\right| \rightarrow \phi>0$ and $\left|q_{z_{n}}\left(\rho_{n}\right)\right| \rightarrow \infty$. Since $q_{t}^{p}(\rho)$ is uniformly bounded, $\phi=\phi^{0}$. Therefore, by (iv) and (v), we have

(viii)

$$
\begin{aligned}
& \phi^{0} x^{\prime}=\phi^{0} x^{\prime 0} \leqq \phi^{0} x \text { for all } x \in X, \\
& \phi^{0} y^{\prime}=\phi^{0} y^{\prime 0} \geqq \phi^{0} y \text { for all }(-k, y) \in Y, \text { or for all } y \in Y_{P} \text {. }
\end{aligned}
$$

The second inequality follows since $q_{t}(\rho)\left(k_{t}(\rho)-k\right)=q_{t}^{P}(\rho)\left(k_{t}(\rho)-k\right)$ is uniformly bounded by the first part of this proof. Since, by the market clearing condition, $y_{t}(\rho)-k_{t}(\rho)=x_{t}(\rho)$, we have

(ix) $y^{\prime 0}=x^{\prime 0}$.

Since, by Sublemma 4, $X \cap Y_{P}$ has a non-empty interior, (ix) contradicts (viii). This completes the proof.

Define $X_{F}^{T}=\left\{\left(x_{1}, \ldots, x_{T}\right) \in X^{T}:\left(-k_{t-1}, y_{t}\right) \in Y, x_{t}=y_{t}-k_{t}, t=1, \ldots, T, k_{0}=\kappa_{0}\right.$, and $\left.k_{T}=0\right\}$, where $X^{T}$ is the Cartesian product of $T X$ 's. We may prove

Sublemma 6. $X^{T} \cap X_{F}^{T}$ has a non-empty interior.

Proof. Due to Lemma 5 of Yano (1984).

We may prove:

Sublemma 7. For each $\varepsilon>0$, there are $0<\rho^{\prime}<1$ and $T \geqq 1$ such that $\rho^{\prime} \leqq \rho<1$ implies $\left|e_{t}(\rho)-e^{\rho}\right|<\varepsilon$ for all $t \geqq T$. 
Proof. By Theorem 3 of Yano (1984).

We now prove Lemma 1.

Proof of Lemma 1. By Sublemma 1, it suffices to show that there are $\beta>0$ and $0<\rho^{\prime}<1$ such that $\rho^{\prime} \leqq \rho<1$ implies $\left|q_{t}(\rho)\right|<\beta$ for all $t \geqq 0$. Let $\varepsilon^{\prime}$ and $\rho_{1}$ be as in Sublemma 3. By Sublemma 7, we may choose $\rho^{\prime} \geqq \rho_{2}$ and $T \geqq 1$ such that $\left|k_{t}(\rho)-k^{\rho}\right|<\varepsilon^{\prime}$ for all $t \geqq T-1$ and all $\rho$ such that $\rho^{\prime} \leqq \rho<1$. Then, by Sublemma 5, $\left|q_{t}(\rho)\right|$ is bounded uniformly in $t \geqq T$ and $\rho$ such that $\rho^{\prime} \leqq \rho<1$. Thus, it sufficies to show that $\left|p_{t}(\rho)\right|$ is bounded uniformly in $\rho$ such that $\rho^{\prime} \leqq \rho<1$ for $t \leqq T-1$. By (iv), we have

$$
\sum_{1}^{T} \rho^{t} W\left(x_{t}(\rho)\right)-\sum_{1}^{T} p_{t}(\rho) x_{t}(\rho) \geqq \sum_{1}^{T} \rho^{t} W\left(x_{t}\right)-\sum_{1}^{T} p_{t}(\rho) x_{t},
$$

for all $\left(x_{1}, \ldots, x_{T}\right) \in X \times \cdots \times X$, where $W(x)=W(x, \gamma(\rho))$. Also by (v), we have that

$$
p_{T}(\rho) y_{T}(\rho)+\sum_{1}^{T} p_{t}(\rho) x_{t}(\rho) \geqq p_{T}(\rho) y_{T}+\sum_{1}^{T} p_{t}(\rho) x_{t},
$$

for all $\left(-k_{t-1}, y_{t}\right) \in Y$ and $x_{t}=y_{t}-k_{t} \in X$ for $t=1, \ldots, T$, with $k_{0}=\kappa_{0}$ and $k_{T}=0$. Suppose that $\left|p_{t}(\rho)\right|$ is not uniformly bounded in $t=1, \ldots, T-1$ and $\rho$ such that $\rho^{\prime} \leqq \rho<1$. Then we may find a sequence $\left\{\rho_{n}\right\}$ with $\rho^{\prime} \leqq \rho_{n}<1$ such that $\quad\left(x_{1}\left(\rho_{n}\right), \ldots, x_{T}\left(\rho_{n}\right)\right) \rightarrow\left(x_{1}^{\prime}, \ldots, x_{T}^{\prime}\right) \quad$ that $\quad\left(p_{1}\left(\rho_{n}\right), \ldots, p_{T}\left(\rho_{n}\right)\right) / \sum_{1}^{T}\left|p_{t}\left(\rho_{n}\right)\right|$ $\rightarrow\left(\phi_{1}, \ldots, \phi_{T}\right)>0$ and that $\sum_{1}^{T}\left|p_{t}\left(\rho_{n}\right)\right| \rightarrow \infty$. Then, by $(\mathrm{x})$, we have that

$$
\sum_{1}^{T} \phi_{t} x_{t}^{\prime} \leqq \sum_{1}^{T} \phi_{t} x_{t}
$$

for all $\left(x_{1}, \ldots, x_{T}\right) \in X \times \cdots \times X$. Since $p_{T}\left(\rho_{n}\right)$ is uniformly bounded, $\phi_{T}=0$. Thus, by (xi), we have

$$
\sum_{1}^{T} \phi_{t} x_{t}^{\prime} \geqq \sum_{1}^{T} \phi_{t} x_{t}
$$

for all $\left(-k_{t-1}, y_{t}\right) \in Y$ and $x_{t}=y_{t}-k_{t} \in X, t=1, \ldots, T$, with $k_{0}=\kappa_{0}$ and $k_{T}=0$. Since, by Sublemma $6, X^{T} \cap X_{F}^{T}$ has a non-empty interior, (xii) and (xiii) lead an obvious contradiction.

By $(v), p_{0}(\rho) \kappa_{0}=p_{1}(\rho) y_{1}(\rho)$. Since $p_{1}(\rho)$ and $y_{1}(\rho)$ are uniformly bounded, $p_{j 0}(\rho)$ is uniformly bounded for $j$ such that $\kappa_{j 0} \neq 0$. Moreover, we may set $p_{j 0}(\rho)=0$ if $\kappa_{j 0}=0$. Thus, Lemma 1 is proved.

Let $\tilde{\rho}$ be as in Sublemma 1. We may prove:

Sublemma 8. There is $\rho_{2}$ such that $\tilde{\rho} \leqq \rho_{2}<1$ and that for each $\varepsilon>0$ the following holds: There is $\delta>0$ such that $\rho_{2} \leqq \rho<1$ and $\left|e_{t}(\rho)-e^{\rho}\right|>\varepsilon$ imply $\Delta_{t}^{\rho}>\delta$. 
Proof. See the proof of Lemma 8 of Yano (1984).

By Lemma 1, we may choose a limit pair $\pi^{1}$ such that there is a sequence $\left\{\rho_{n}\right\}$ such that $\rho_{n} \rightarrow 1$ and that $\pi^{\rho_{n}}$ converges to $\pi^{1}$, where $\pi^{\rho_{n}}, n=1,2, \ldots$, are those chosen at the beginning of this section. Denote $\pi^{1}=(e(\rho), p(1)$ $\left.e^{*}, q^{*}: \gamma(1)\right), \underset{e}{e(1)}=\left\{e_{t}(1)\right\}, e_{t}(1)=\left(\left(-k_{t-1}(1) y_{t}(1)\right), x_{t}^{1}(1), \ldots, x_{t}^{I}(1)\right), \underset{\sim}{p(1)}=\left\{p_{t}(\tilde{1})\right\}$, $e^{*}=\left(\left(-k^{*}, y^{*}\right), x^{1 *}, \ldots, x^{I *}\right)$. We will prove:

Sublemma 9. $e_{t}(1) \rightarrow e^{*}$ as $t \rightarrow \infty$.

Proof. Since $e_{t}\left(\rho_{n}\right) \rightarrow e_{t}(1), e^{\rho_{n}} \rightarrow e^{*}$, and $\gamma\left(\rho_{n}\right) \rightarrow \gamma(1)$ as $\rho_{n} \rightarrow 1$, the definition of $A_{t}^{\rho}$ implies that $A_{t}^{\rho_{n}}$ converges to some non-negative, say, $\Delta_{t}^{*}$ for all $t$. Therefore, by Sublemma 8 , for any $\varepsilon>0$ there is $\delta>0$ such that $\left|e_{t}(1)-e^{*}\right|>\varepsilon$ implies $\Delta_{t}^{*}>\delta$. By Sublemma 2, we have a subsequence of $\left\{\rho_{n}\right\}$, call it $\left\{\rho_{n}\right\}$ again, such that $\Lambda_{0}^{\rho_{n}}$ converges. Denote the limit as $\Lambda_{0}^{*}$. Since $\Delta_{t}^{\rho_{n}} \rightarrow \Delta_{t}^{*}$, eq. (ii) implies that, by induction, $\Lambda_{t}^{\rho_{n}} \rightarrow \Lambda_{t}^{*}$ for all $t$. Note that since $\Lambda_{t}^{\rho_{n}} \geqq 0, \Lambda_{t}^{*} \geqq 0$. Then, by (ii), $A_{t-1}^{*}-\Lambda_{t}^{*}=\Delta_{t}^{*}$ for all $t$. Thus, since $\Lambda_{t}^{*} \geqq 0$ for all $t$,

(xiv) $\quad \Lambda_{0}^{*} \geqq \sum_{t=1}^{T} \Delta_{t}^{*}$,

for all $T$. Suppose that there is $\varepsilon>0$ such that $\left|e_{t}(1)-e^{*}\right|>\varepsilon$ for infinitely many $t$ 's. Then, as is noted above, $\Delta_{t}^{*}>\delta$ for some $\delta>0$ for infinitely many $t$ 's. Since $A_{t}^{*} \geqq 0$ for all $t$, this implies $\sum_{t=1}^{T} \Delta_{t}^{*} \rightarrow \infty$, which contradicts (xiv). Thus, $e_{t}(1) \rightarrow e^{*}$. This completes the proof.

We now prove Lemma 4.

Proof of Lemma 4. Take a feasible allocation path from $\sum_{i} \kappa_{0}^{I}$, where $\left(\kappa_{0}^{1}, \ldots, \kappa_{0}^{I}\right)$ is the initial allocation of capital chosen above. Denote this path as $\underline{e}=\left((-\underset{\sim}{k}, y), \underline{x}^{1}, \ldots, \underline{x}^{I}\right),(-\underset{v}{k}, y)=\left\{\left(-k_{t-1}, y_{t}\right)\right\}$, and $\underline{x}^{i}=\left\{x_{t}^{i}\right\}, i=1, \ldots, I$. Let $x_{t}(1)=\sum_{i} x_{t}^{i}(\tilde{1})$ and $x_{t}=\sum_{i} x_{t}^{i}$. Without loss of generality, we may assume $y_{t}-k_{t}=x_{t}$ for all $t \geqq 1$. Since $\pi^{\rho_{n}}$ converges to $\pi^{1}$, inequalitics (iv) and (v) imply

$$
W\left(x_{t}(1)\right)-p_{t}(1) x_{t}(1) \geqq W\left(x_{t}\right)-p_{t}(1) x_{t}, \quad \text { and }
$$

$$
0=p_{t}(1) y_{t}(1)-p_{t-1}(1) k_{t-1}(1) \geqq p_{t}(1) y_{t}-p_{t-1}(1) k_{t-1} \text {, }
$$

where $W(x)=W(x, \gamma(1))$. Define $\delta_{t}=W\left(x_{t}(1)\right)-p_{t}(1) x_{t}(1)-\left(W\left(x_{t}\right)-p_{t}(1) x_{t}\right)+$ $\left.p_{t}(1) y_{t}(1)-p_{t-1}(1) k_{t-1}(1)-\left(p_{t}(1) y_{t}-p_{t-1}(1) k_{t-1}\right)\right)$. Then, by (xiv) and (xv), $\delta_{t} \geqq 0$ for all $t \geqq 1$. Note, by Sublemma $9, e_{t}(1) \rightarrow e^{*}$. Thus, by a method similar to that used to prove Sublemma 8 , we may find $T \geqq 1$ such that the following holds. For any $\varepsilon>0$ there is $\delta>0$ such that $\left|e_{t}(1)-e_{t}\right|>\varepsilon$ and $t \geqq T$ imply $\delta_{t}>\delta$. Note $k_{0}(1)=\kappa_{0}\left(=\sum_{i} \kappa_{0}^{i}\right), y_{t}-k_{t}=x_{t}$, and $y_{t}(1)-k_{t}(1)=x_{t}(1)$, where the last inequality follows since, by (11.iv), $y_{t}\left(\rho_{n}\right)-k_{t}\left(\rho_{n}\right)=x_{t}\left(\rho_{n}\right)$. Therefore, the definition of $\delta_{t}$ implies 


$$
\sum_{t=1}^{T}\left(W\left(x_{t}\right)-W\left(x_{t}(1)\right)\right)=p_{T}(1)\left(k_{T}(1)-k_{T}\right)-\sum_{t=1}^{T} \delta_{t}
$$

If $p_{T}(1)\left(k_{T}(1)-k_{T}\right) \rightarrow 0$ as $T \rightarrow \infty$, (xvi) implies $\sum_{t=1}^{\infty}\left(W\left(x_{t}\right)-W\left(x_{t}(1)\right)=\right.$ $-\sum_{t=1}^{\infty} \delta_{t} \leqq 0$. If $p_{t}(1)\left(k_{T}(1)-k_{T}\right) \nrightarrow 0,\left|e_{t}(1)-e_{t}\right|>3$ for some $\varepsilon>0$ for infinitely many $t$ 's. Then, as noted above, $\delta_{t}>\delta$ for some $\delta>0$ for infinitely many $t$ 's. Then, $\sum_{t=1}^{T} \delta_{t} \rightarrow \infty$ as $t \rightarrow \infty$. By Sublemma $1,\left|p_{T}(1)\right|$ and $\left|k_{T}(1)\right|$ are bounded uniformly in $T$. Moreover, we may prove that any feasible path $\left\{e_{i}\right\}$ is bounded uniformly in $t$ [see Yano (1984), Lemma 3]. Thus, since $p_{T}(1)\left(k_{T}(1)-k_{T}\right)$ is bounded uniformly in $T$, and since $\sum_{t=1}^{T} \delta_{t} \rightarrow \infty$, (xvi) implies $\sum_{t=1}^{T}\left(W\left(x_{t}\right)-W\left(x_{t}(1)\right)\right) \rightarrow-\infty$. This proves Lemma 4 .

Lemmas 2 and 3 require Assumptions (a.1) and (a.2), and are extensions of Sublemmas 7 and 9. Whereas the stability of only allocation paths is shown in the sublemmas, the lemmas assert that of price paths as well. Since Assumptions (a.1) and (a.2) simply require that the indifference surfaces of a consumer and the boundary of the production set are smooth, we may prove Lemmas 2 and 3 by routine methods. Proofs are therefore omitted.

\section{References}

Arrow, K. and G. Debreu, 1954, Existence of equilibrium in a competitive economy, Econometrica $27,265-290$.

Arrow, K. and F. Hahn, 1971, General competitive analysis (Holden-Day, San Francisco, CA).

Atsumi, Hiroshi, 1965, Neoclassical growth and the efficient program of capital accumulation, Review of Economic Studies 32, 127-136.

Becker, Robert, 1980, On the long run steady state in a simple dynamic model of equilibrium with heterogeneous households, Quarterly Journal of Economics, 375-382.

Bewley, Truman, 1972, Existence of equilibria in economics with infinitely many commodities, Journal of Economic Theory 4, 514-540.

Bewley, Truman, 1982, An integration of equilibrium theory and turnpike theory, Journal of Mathematical Economics 10, 233-267.

Brock, William, 1970, On existence of weakly maximal programmes in a multi-sector economy, Review of Economic Studies 37, 275-280.

Brock, William and J. Scheinkman, 1976, Global asymptotic stability of optimal control system with applications to the theory of economic growth, Journal of Economic Theory 12, 164190.

Cass, D. and K. Shell, 1976, The structure and stability of competitive dynamic systems, Journal of Economic Theory 12, 31-70.

Debreu, Gerard, 1959, Theory of value (Wiley, New York).

Gale, David, 1976, On optimal development in a multi-sector economy, Review of Economic Studies 34, 1-18.

Hicks, John, 1939, Value and capital (Oxford University Press, Oxford).

Malinvaud, Edmond, 1953, Capital accumulation and efficient allocation of resources, Econometrica 21, 233-268.

McKenzie, Lionel, 1959, On the existence of general equilibrium for a competitive market, Econometrica 27, 54-71.

McKenzie, Lionel, 1974, Turnpike theorems with technology and welfare function variable, in: J. Łos and M.W. Eos, eds., Mathematical models in economics (Elsevier, New York).

McKenzie, Lionel, 1976, Turnpike theory. Econometrica 44, 841-865. 
McKenzie, Lionel, 1979, Optimal economic growth and turnpike theorems, Discussion paper no. 79-1 (University of Rochester, Rochester, NY) in: K. Arrow and M. Intrigligator, eds., Handbook of Mathematical Economics (North-Holland, New York) forthcoming.

McKenzie, Lionel, 1981, The classical theorem on existence of competitive equilibrium, Fconometrica 49, 819-842.

Negishi, T., 1972, General equilibrium theory and international trade (North-Holland, Amsterdam).

Peleg, B. and H. Ryder, 1974, The modified golden rule of a multi-sector economy, Journal of Mathematical Economics 21, 193-198.

Rader, Trout, 1971, The economics of feudalism (Gordon and Breach, New York).

Radner, Roy, 1961, Paths of economic growth that are optimal with regard only to final state, Review of Economic Studies 28, 98-104.

Ramsey, Frank, 1928, A mathematical theory of saving, Economic Journal 38, 543-559.

Rockafellar, R. T., 1976, Saddle points of Hamiltonian systems in convex Lagrange problems having a nonzero discount rate, Journal of Economic Theory 12, 71-113.

Rudin, W., 1976, Principle of mathematical analysis (McGraw-Hill, New York).

Scheinkman, José, 1976, On optimal steady states of $n$-sector growth models when utility is discounted, Journal of Economic Theory 12, 11-20.

Sutherland, W., 1970, On optimal development in a multi-sectoral economy: The discounted case, Review of Economic Studies 37, 585-589.

Takayama, Akira, 1974, Mathematical economics (Dryden, Hinsdale, IL).

Weitzman, M.L., 1973, Duality theory for infinite horizon convex models, Management Science $19,783-789$.

Von Weizsäcker, C.C., 1965, Existence of optimal programs of accumulation for an infinite time horizon, Review of Economic Studies 32, 85-104.

Yano, Makoto, 1981a, Competitive equilibria on turnpikes in a McKenzie economy, Ph.D. dissertation, Ch. 3 (University of Rochester, Rochester, NY).

Yano, Makoto, 1984b, Competitive equilibria on turnpikes in a McKenzie economy, II: An asymptotic turnpike theorem, Mimeo. (Cornell University, Ithaca, NY).

Yano, Makoto, 1984, Competitive equilibria on turnpikes in a McKenzie economy, I: A neighborhood turnpike theorem, Mimeo. (Cornell University, Ithaca, NY). 